\title{
The Philosophy of Interdisciplinarity: Sustainability Science and Problem-Feeding
}

\author{
Henrik Thorén · Johannes Persson
}

Published online: 17 November 2013

(c) The Author(s) 2013. This article is published with open access at Springerlink.com

\begin{abstract}
Traditionally, interdisciplinarity has been taken to require conceptual or theoretical integration. However, in the emerging field of sustainability science this kind of integration is often lacking. Indeed sometimes it is regarded as an obstacle to interdisciplinarity. Drawing on examples from sustainability science, we show that problem-feeding, i.e. the transfer of problems, is a common and fruitful-looking way of connecting disparate disciplines and establishing interdisciplinarity. We identify two species of problem-feeding: unilateral and bilateral. Which of these is at issue depends on whether solutions to the problem are fed back to the discipline in which the problem originated. We suggest that there is an interesting difference between the problem-feeding approach to interdisciplinarity and the traditional integrative perspective suggested by among others Erich Jantsch and his colleagues. The interdisciplinarity resulting from problem-feeding between researchers can be local and temporary and does not require collaboration between proximate disciplines. By contrast, to make good sense of traditional integrative interdisciplinarity we must arguably associate it with a longer-term, global form of close, interdisciplinary collaboration.
\end{abstract}

Keywords Interdisciplinarity $\cdot$ Problem-feeding $\cdot$ Jantsch ·

Sustainability science

\footnotetext{
H. Thorén $(\bowtie)$

Department of Philosophy, Lund University, Lundagard, 22222 Lund, Sweden

e-mail: henrik.thoren@fil.lu.se

J. Persson

Lund University Center of Excellence for Integration of Social and Natural Dimensions of

Sustainability (LUCID), P.O. Box 170, 22100 Lund, Sweden

e-mail: johannes.persson@fil.lu.se
} 


\section{Interdisciplinarity: A Traditional Perspective}

One day you are in, and the next you are out. The term 'interdisciplinarity' passes in and out of fashion. However, the phenomenon itself is associated with fundamental issues in science and philosophy.

Philosophers of science who have studied interdisciplinarity, whether or not they deploy the term itself, often focus on relationships obtaining between proximate disciplines or fields. For instance, since the 1970s the life sciences have been a prime source of material, and numerous case studies have been made showing how our knowledge can grow, and how discoveries can be made, when fields such as biochemistry and cell biology interact.

However, interdisciplinary collaboration can involve less proximate disciplines as well. This is one reason why we want to look more closely, from a philosophy of science perspective, at a new kind of interdisciplinary case, namely sustainability science.

The other reason for focusing on sustainability science is this. It is not clear that traditional accounts of interdisciplinarity capture a certain type of interdisciplinary collaboration that we believe is characteristic of sustainability research: the transfer of problems (and sometimes solutions) from one discipline to the other. We call this type of interdisciplinarity 'problem-feeding'.

This article starts by presenting the traditional perspective and the difficulties it has handling the interdisciplinary field of sustainability science. Then we introduce the notion of problem-feeding and compare it with recent discussion of the philosophy of interdisciplinarity. We discuss two varieties of problem-feeding: unilateral and bilateral. Which of these is at issue depends on whether solutions to the problem are fed back to the discipline in which the problem originated.

Since the early 1970s, and certainly since the publication of Jantsch's (1970/1972), the type and degree of conceptual or, broadly speaking, theoretical integration of the participating disciplines has been the primary basis on which to sort types of interdisciplinary encounter. This focus on conceptual or theoretical relationships defines what might be called the traditional perspective on interdisciplinarity.

Figure 1 illustrates the traditional view. Jantsch characterizes multidisciplinarity in terms of "no cooperation", interdisciplinarity in terms of "coordination by higher-level concept", and transdisciplinarity in terms of "multilevel coordination of entire education/ innovation system". At other places he writes about multidisciplinarity as a situation with a variety of disciplines whose relationships have not been made explicit. The kinds of relationship he and others belonging to the traditional perspective are talking about, then, are conceptual or theoretical relationships.

Often multidisciplinarity is still understood as non-integrative, or additive, amounting merely to the juxtaposition of knowledge claims from different sources; correspondingly, interdisciplinarity is quite frequently thought to involve more 'internal' integration than multidisciplinary approaches do (Klein, 1990, 56-58).

An additional feature of Jantsch's approach is "the basic evolutionary ladder" (Jantsch 1970/1972, 15). Collaboration may start as multidisciplinary and end as transdisciplinary. The three categories we referred to in Fig. 1 are steps for climbing up the basic evolutionary ladder. Many advocates of the traditional perspective have a similar view. For instance, Jantsch observes that another influential writer on interdisciplinarity, Piaget, conceives of inter- and transdisciplinarity as the two highest steps "on a rigid ladder of levels" (ibid., 18). What happens in the step Jantsch calls interdisciplinary is especially important. With this step: 
Fig. 1 The traditional perspective. Source Figure adapted from Jantsch (Jantsch 1970/1972, 15)

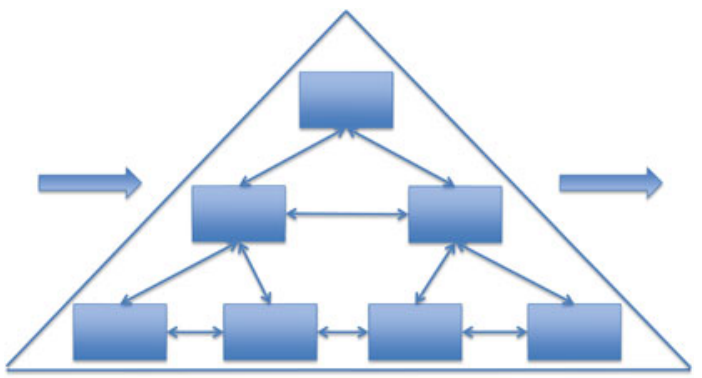

Transdisciplinarity: multilevel coordination...

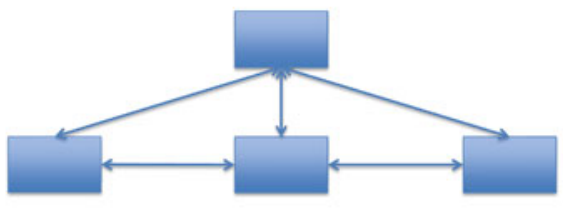

Interdisciplinarity: coordination by higherlevel concept

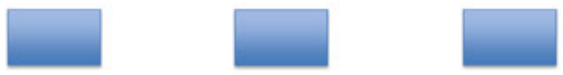

Multidisciplinarity: no cooperation

A common axiomatics for a group of related disciplines is defined at the next higher hierarchical level, thereby introducing a sense of purpose. (Jantsch 1970/1972, 16)

Jantsch's view that the interdisciplinary step necessarily introduces an effective sense of purpose is somewhat less widely shared, and therefore it should not be included in the traditional perspective. The sense of purpose allows us to answer the question "interdisciplinarity to what end?" and influences the disciplines. In Jantsch's view, with the introduction of interdisciplinary relations the linked scientific disciplines change in respect of their concepts, structures, and aims.

Transdisciplinarity is then taken to involve massive coordination on the basis of a generalized axiomatics. Jantsch's idea is that several (two-level) interdisciplinary systems are linked, and that changes in overall system goals have an impact on concepts and principles throughout all of the linked, interdisciplinary scientific disciplines. If we bracket Jantsch's insistence on change being effected from above, his conception of transdisciplinarity is part of the traditional perspective. For instance, in Apostel et al. (1972, 25f) transdisciplinarity is defined as the ordering of disciplines under a common set of axioms.

Jantsch's idea that interdisciplinary collaboration evolves from multidisciplinarity to transdisciplinarity is clearly visible in contemporary thinking about interdisciplinary science, and in particular in sustainability science. Consider, for example, the terms in which a quite large Swedish research programme, Lund University Centre of Excellence for Integration of Social and Natural Dimensions of Sustainability (LUCID), explains how collaboration within the programme will develop over a period of 10 years (Fig. 2).

At its inception, in 2008, LUCID engaged researchers from seven disciplines and four faculties. Although the researchers were officially working on joint research problems, such as land-use change, the problems were approached from each individual's 


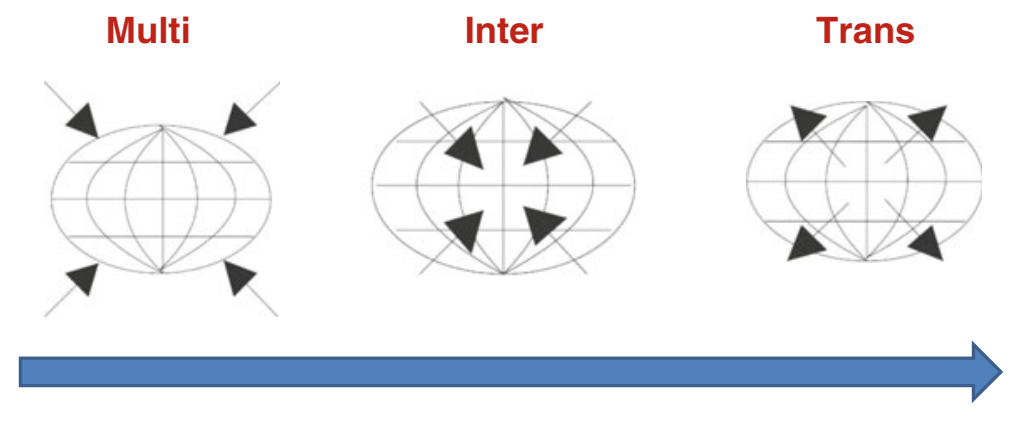

Ten years

Fig. 2 Interdisciplinarity over time. Source Figure adapted from Jerneck et al. $(2011,79)$

disciplinary perspective. A few years have passed since 2008, and according to the plan the current interdisciplinary phase will be "resulting in a co-evolution of theories for sustainability science"; finally, in the transdisciplinary phase, "theories evolve and mature to gradually incorporate more domains and transcend the boundary between science and practice" (Jerneck et al. 2011, 79). The conceptual, or theoretical, integration in the interdisciplinary phase is reminiscent of the picture painted by Jantsch. The assumption that the programme could not move forward at a faster pace probably accords with common sense. Integration by higher-level concepts and theories takes time. If such integration is needed for 'higher' levels of interdisciplinarity, such as transdisciplinarity, to develop, higher levels of interdisciplinary collaboration plausibly occur after less advanced ones.

We deploy sustainability science as a test case against which accounts of interdisciplinarity can be evaluated. It clearly merits consideration in its own right. We also think it can be used to assess perspectives such as Jantsch's. Sustainability science raises interdisciplinary issues of a kind Jantsch would surely have wanted to be able to account for: "In most general terms, the purpose of the university may be seen in the decisive role it plays in enhancing society's capability for continuous self-renewal" (Jantsch 1970/1972, 12). Given this, the field of sustainability science cannot be said to be unrepresentative in the traditional perspective.

\section{The Need for an Alternative to the Traditional Perspective}

Despite its somewhat unusual amplification of a sense of purpose as an essential component of interdisciplinarity, Jantsch's account might appeal to many philosophers of science. Its focus on integration by concept or theory is well-known from the unity of science movement, from Nagelian reductionism (Nagel 1961), and from other less reductionist accounts. We discuss some of the modern varieties of this view in Sect. 7 below. In this section we highlight two problems with the traditional perspective.

(2a) Pretty clearly, the traditional perspective is threatened by Jantsch's treatment of the basic evolutionary ladder. It begins to look like a rather ineffective tool for characterizing interdisciplinary collaborations of shorter duration. Sustainability projects aimed at practical problem-solving are frequently of this type. The traditional perspective becomes incapable of discriminating between shorter projects, evaluating them all as 
multidisciplinary endeavours involving no cooperation, when surely these collaborations might differ in ways that a philosophy of interdisciplinarity wants to be able to account for.

As a potential counterexample to Jantsch's and our view (that traditional interdisciplinarity takes plenty of time to establish), it should be noted that there is at least one popular account of transdisciplinarity in which Jantsch's conceptual and theoretical integration is assumed to take place in the absence of previous stages of interdisciplinary collaboration. Michael Gibbons and Helga Nowotny and their colleagues attracted considerable attention by claiming that modern science often involves transdisciplinary knowledge generation in "Mode 2" (Gibbons et al. 1994; Nowotny et al. 2001). The Mode 2 concept is designed to suit enquiries in which researchers from different disciplinary perspectives come together to work on problems "a context of application." The Mode 2 concept of transdisciplinarity deployed here is similar to Jantsch's concept of transdisciplinarity in many ways, but it does not contain his additional assumption that before (Mode 2) transdisciplinarity the research team first spends time in less integrated forms of interdisciplinary collaboration. Instead, a possibly unique theoretical framework is generated from scratch, partly as a result of negotiations in rather heterogeneous, local research groups.

Does the Mode 2 account encourage confidence in the idea that the traditional perspective on interdisciplinary research can be successfully applied to projects of shorter duration, and to more practically oriented projects as well? Not really. What it establishes is merely that there is no contradiction involved in assuming that interdisciplinary research can be integrated by a theoretical framework that has been designed and agreed upon in a local interdisciplinary context. But examples of Mode 2 projects in sustainability science that are both practically oriented and result in new theoretical frameworks are rare.

Whether or not one believes in the basic evolutionary ladder, this already suggests the need for a complementary account of interdisciplinarity-one that does not build on integration by concept or theory.

(2b) The case of sustainability science highlights an independent shortcoming, or potential shortcoming at any rate, of the traditional account of interdisciplinarity. Some sustainability scientists who are committed to the long-term project do not regard themselves as promoters of integration by concept or theory. For instance, responding to a questionnaire, a senior research scientist from a European centre for sustainable development research wrote to us:

We don't use the label 'sustainability science', since we define ourselves primarily as focusing on themes, but from different disciplinary backgrounds. We are very cautious about defining our work and approach as being part of a new discipline, since this closes off doors that need to remain open for inter- and transdisciplinarity.

On our interpretation, the response conveys two things of importance. The first is that the background disciplines the research scientist is thinking of will not themselves be theoretically integrated as a result of the thematic work. As some areas of sustainability research illustrate, it is easy to locate contexts of interdisciplinary collaboration which are unlikely ever to involve integration "by higher-level concept" - contexts, moreover, in which time is not the issue. Certain disciplines are rather rigid, in the sense that they are unlikely to change conceptually or theoretically (Jantsch 1970/1972, 19), especially as a result of interdisciplinary collaboration. Many reasons for rigidity can be identified (Persson and Sahlin 2013, Ch. 10). One possibility is that a powerful disciplinary matrix is already in place (Kuhn 1969/1970) within one, or both, of the involved disciplineseffectively blocking theoretical integration of the pair. And in some cases the conceptual 
distance might simply be too big for such integration to be possible (e.g. see Edlund et al. 1986, 24). Thus, one might wonder whether the integration of natural and social dimensions of sustainability could ever mean that the natural and social sciences should be conceptually or theoretically integrated. This is the factual problem. Conceptual or theoretical integration of distant or rigid disciplines is unlikely.

In such cases are we then forced to conclude that we are at a stage of multidisciplinarity, i.e. in a context with no cooperation? Various things might happen even if the existing natural and social sciences do not change. For instance, new disciplines are sometimes born. This is the second, and the main, worry expressed in the quotation. The National Science Foundation has inherited the slogan Today's interdisciplinarity, tomorrow's disciplinarity. This is what the research scientist we have quoted wants to avoid. She sees a normative problem with this alternative. Sustainability research should not lead to a new discipline: a distinct theoretical and conceptual framework might weaken the interdisciplinary character of the field. But there is a factual problem with the idea as well. For if sustainability science emerges as a new discipline, will it integrate concepts and theoretical fragments from, say, political science and oceanography? Again, the conceptual distance between the two background theories might simply be too great to allow such integration.

As our case study, sustainability science, shows, a complementary perspective is required if we are to understand interdisciplinary collaboration in contexts where coordination by higher-level concepts does not take place. This paper aims to develop this perspective.

\section{Two Examples from Sustainability Science}

Sustainability science is a fairly young field. It was consolidated as an international science policy project in the preparations for the World Summit on Sustainable Development in Johannesburg in 2002 (Jerneck et al. 2011, 70). We want to present two examples with a bearing on sustainability issues-one contemporary, the other from a time well before the birth of sustainability science.

(3a) In sustainability science problems are frequently defined by natural sciences and then exported to social science. This is because sustainability challenges are often identified initially by the natural sciences and subsequently communicated to society as potential (future) events or states of the world that will cause societal problems that we have not met with before. In an influential paper in the Proceedings of the National Academy of Sciences of the United States of America (PNAS) Timothy Lenton et al. (2008) defined and identified a number of climatic tipping elements (and a few tipping points).

These tipping elements are components of the earth's climate system which, once pushed across a certain threshold, or tipping point, are likely to exhibit non-linear, disruptive change. The PNAS text lists 15 policy-relevant tipping elements, including Arctic summer sea-ice, the Greenland ice sheet, Atlantic thermohaline circulation, and the Indian summer monsoon.

The authors argue that these elements can be pushed by human interaction across a tipping point resulting in Arctic sea-ice loss, the melting of the Greenland ice sheet, Atlantic deep water formation, Indian monsoon chaotic multi-stability, and so on. Furthermore, every element contributes significantly to human welfare as we know it today.

In many cases the identification of these tipping points clearly falls within the domain of one or other of the natural science disciplines, or an aggregate of them. Lenton et al. (2008) focus exclusively on tipping elements that trigger a qualitative change taking place within 
an "ethical time horizon" (revolving around the idea that events or states should not be too distant in time to be politically significant). However, even with this commitment to societal issues the task of identifying such events or states does not require real interdisciplinary engagement. On the other hand, this is as far as natural science seems to getinitially - in approaching the societal dimension. For instance, the task of addressing the issues of mitigation or adaptation is first and foremost one for social science.

Other problems that Lenton and his colleagues consider are more specific and hinge on the applicability of their own notion of a tipping element. For instance, they are interested in the question whether tipping elements in the social-economic system can be identified.

(3b) Scientific investigation of the carbon cycle and its role in climate change has been of interest for a long time now. Before the 1950s research concentrated on the question whether changes in atmospheric $\mathrm{CO}_{2}$ concentrations explained the occurrence of ice-ages. However, since then sustainability issues have often been in focus. Nowadays, mapping out the way carbon cycles through the earth system is a highly interdisciplinary affair involving physics, chemistry, ecology, biology and economics. Towards the end of the nineteenth century, however, the carbon cycle and the temperature effects of $\mathrm{CO}_{2}$ were the concern of physicists. We select one of the most important breakthroughs in this line of research as our illustration.

$\mathrm{CO}_{2}$ was known to have 'greenhouse' properties early on. Both Joseph Fourier, in the 1820 s, and John Tyndall, in the 1860s, had been interested in the properties of the gas (see Weart 2003, 2007), and as early as 1896 Arrhenius produced a quantitative model of the impact of different atmospheric concentrations of $\mathrm{CO}_{2}$ on the mean surface temperature of the earth. His results suggested that doubling the concentration of $\mathrm{CO}_{2}$ would result in mean temperatures rising by approximately $5-6{ }^{\circ} \mathrm{C}$.

If $\mathrm{CO}_{2}$ concentrations had indeed varied to this extent, those variations would potentially have explained why ice ages occur. However, it was not known whether such variation was possible. Arrhenius' model had not included the role of the oceans in $\mathrm{CO}_{2}$ dynamics. At the time this role was not well understood. It was known that plain water dissolves $\mathrm{CO}_{2}$, so there was a suspicion that the variability of atmospheric $\mathrm{CO}_{2}$ concentration was comparatively low. It was thought possible that any increase would quickly be neutralised, as the oceans would absorb the surplus. Arrhenius himself notes this possibility in the 1896 paper in which his model is formulated. His suspicion was widely retained for the next five decades.

In 1957 Craig $(1957,2)$ noted that the range of "[r]ecent estimates of the residence time of a molecule of carbon dioxide in the atmosphere, before entering the sea, range from $16 \mathrm{~h}$ (Dingle 1954) to the order of 1,000 years" (Plass 1956) (see also Weart 1997). In other words, in 1957 it was not known if the oceans' absorptive properties would cancel out increases in $\mathrm{CO}_{2}$ concentrations within a timeframe ensuring that $\mathrm{CO}_{2}$ was not a cause of climate change.

Against this background a central problem became that of understanding how $\mathrm{CO}_{2}$ cycles through the atmosphere and the oceans. Improved knowledge of the absorptive properties of the oceans - especially-would shed light on the mechanisms governing the variability of $\mathrm{CO}_{2}$ concentrations in the atmosphere. An understanding of this would not only be essential to the solution of the old problem of the occurrence of ice ages. It would help to explain the potential dangers of emitting large quantities of fossil carbon into the atmosphere.

The first, and perhaps most vital, step towards a solution to this problem was provided by two researchers at the Scripps Institution of Oceanography in La Jolla, California. One of them, the oceanographer Roger Revelle, was the head of the institute. The other, Hans 
Süess, was a chemist and specialist in carbon dating. In 1957 they published a joint paper in which they calculate the average time a $\mathrm{CO}_{2}$ molecule spends in the atmosphere. Towards the end of the paper they note that they have used a simplified model of the ocean. They argue that this simplification is likely to affect their results as a consequence of two interconnected mechanisms. One concerns the chemical properties of the oceans; the other has to do with their mechanical properties. First, oceans act as so-called buffer solutions. They will resist changes in $\mathrm{pH}$ such as those induced by the absorption of $\mathrm{CO}_{2}$. In the surface water two equilibrium reactions realise this mechanism. One is between aqueous carbon dioxide and carbonic acid $\left(\mathrm{H}_{2} \mathrm{CO}_{3}\right)$; the other is between carbonate ions $\left(\mathrm{CO}_{3}{ }^{2-}\right)$ and bicarbonate ions $\left(\mathrm{HCO}_{3}{ }^{-}\right)$. As $\mathrm{CO}_{2}$ is dissolved in oceanic water these equilibrium reactions are affected. The result is that even if most of the $\mathrm{CO}_{2}$ is initially dissolved, it will be evaporated right back into the atmosphere. An increase in the $\mathrm{CO}_{2}$ content of the atmosphere of $10 \%$ is balanced by as little as a $1 \%$ in the total $\mathrm{CO}_{2}$ content of the ocean (Bolin and Eriksson 1959, 131). This brings us to the second mechanism. Revelle and Süess (1957) treat the ocean as "one well-mixed reservoir of $\mathrm{CO}_{2}$ ". This assumption is problematic, they note, as the oceans are layered. While oceanic water mixes quite quickly in the horizontal dimension within layers, it mixes excruciatingly slowly between them.

Our current understanding is that the vertical mixing of layers is a process that takes place over millennia. The implication is that oceans, in the short and medium term, do not act to dissolve $\mathrm{CO}_{2}$ in any important way. Hence the oceans do not balance $\mathrm{CO}_{2}$ concentrations in the atmosphere over relevant timeframes, nor do they preclude $\mathrm{CO}_{2}$ from playing a role in climate change.

One way to interpret this piece of history is as follows. The task here was to provide an account of how the oceans function with respect to $\mathrm{CO}_{2}$. To provide such an account two mechanisms on different scales needed to be coupled. One of these mechanisms belonged to the domain of oceanography, and the other belonged to chemistry. The role of the oceans could not be modelled until knowledge from oceanography and chemistry had been integrated.

Now, both cases we have presented involve the exporting of problems, but in the second case that export is only part of the story. What is equally, or more, important in the $\mathrm{CO}_{2}$ example is the importing of solutions. This process of involving more and more disciplines in the importing of solutions has continued. Notably, biological material provides a gigantic reservoir of carbon offering opportunities for a range of life science disciplines to contribute to our understanding of the $\mathrm{CO}_{2}$ cycle.

\section{Interdisciplinary Types, and Kinds of Interdisciplinary Relata}

We began by presenting the traditional perspective. The interdisciplinary stories we have just told about sustainability science would perhaps have been judged as narratives in which there is no cooperation of the Jantschian sort. In the first case, (3a), real interdisciplinary engagement is not even required. In the second case, (3b), there is clearly cooperation, but it is cooperation without coordination by new higher-level concepts. However, in the traditional perspective as we have presented it coordination by higherlevel concept is crucial, and therefore neither of our two sustainability examples qualifies as a case of interdisciplinarity as that has traditionally been conceived. At the same time both examples are clearly interdisciplinary in another sense, and a satisfactory account of interdisciplinarity should recognize this. 
Before we introduce our own suggestion as to how to understand interdisciplinarity in (3a) and (3b) we want briefly to take note of the fact that a conception of interdisciplinarity more pluralistic than one focusing exclusively on coordination by concept or theory is implicit in many traditional accounts. For instance, Apostel et al. (1972, 25) speak of (advanced forms of) interdisciplinarity as activities involving the integration of concepts, methodology, procedures, epistemology, terminology and data. So the traditional perspective recognizes additional kinds of interdisciplinary relata.

It is possible, of course, to be more relaxed than Jantsch about what type of constituent is the more fundamental for climbing the basic evolutionary ladder. Facilitating Interdisciplinary Research characterizes interdisciplinary research in a much more relaxed way as a mode of research that:

integrates information, data, techniques, tools, perspectives, concepts, and/or theories from two or more disciplines or bodies of specialized knowledge to advance fundamental understanding or to solve problems whose solutions are beyond the scope of a single discipline or area of research practice. (National Academy of Sciences et al. 2005, 39)

An alternative approach to the traditional one would be to bring order to the often muddled picture we have of interdisciplinarity by sorting interdisciplinary projects, or aims, by the types of constituent that interact in encounters between researchers of the disciplines involved (Thorén and Persson 2011). The types of constituent stated in Apostel et al. (1972) and (National Academy of Sciences et al. 2005) could indeed provide the starting point for such an endeavour. However, before we begin sorting interdisciplinary kinds by the types of constituent suggested in lists such as those above, we might ponder whether they are exhaustive, or whether any salient types of constituent are missing.

\section{Transfer of Problems}

Our conception of interdisciplinary research in this article is pluralistic, but it is not identical with the conceptions we touched upon in the last section. For one thing, we invoke a type of constituent not mentioned in the passage excerpted above. We also make use of a relation other than integration. We do not challenge the idea that the traditional perspective is sometimes applicable. We simply deny that it always is.

Let us look back at the two examples involving sustainability issues - the tipping-points and the $\mathrm{CO}_{2}$ cycle. What we claim to be one important feature these two cases have in common is that problems in them are moved from one discipline to another. Lenton et al. (2008) can be taken as typical of a development within sustainability science that has been going on for a decade or more. Many of the initial results concerning the impacts of climate change have been delivered by various natural science disciplines. New problems have been created by these results, many of which do not appear to be natural science problems at all. Some, of course, are quite simply practical problems for the political and social institutions involved, but many are scientific problems. The impacts of climate change can only partly be understood from the perspective of climate science. Sea-level rise, or drastic changes in the weather patterns, will alter the conditions under which our social structures have been formed. This will obviously affect those structures, but how? What will happen to people living in areas that slowly become less habitable? Arrhenius was interested in the impact of climate change on human societies. He thought a warmer climate would result in better agriculture and a more pleasant and easier life, especially for poor northerners. 
Lenton and colleagues wonder about how to prevent us from passing the tipping points. But these questions and problems are transferred to sciences better capable of researching them. The problem-feeding character of interdisciplinarity might be especially clear in sustainability science, with its focus on how new social problems emerge as a result of changes in the natural world. The examples from Lenton and his colleagues are of this type.

\subsection{Unilateral Problem-Feeding}

Problem-feeding in sustainability science is sometimes unilateral. It is problem-feeding, as it were, without solution-feeding. In general, one field (or discipline) may rely on another as a source of problems; these problems may or may not be problems for the discipline in which the problem originally emerged. To shift to an almost trivial example, consider the relationship between philosophy of science and science. Clearly the philosophy of, say, physics, relies on physics (the discipline) for some of its problems. What happens in physics is part of the subject matter of philosophy of physics. However, the problems may not be problems for physicists, and their resolution may have negligible impact on the way physicists conduct their research. We do not mean to take any particular stance on this question. We simply highlight the fact that if one discipline relies on another for its problems-in full, or in part-this does not necessarily mean that the discipline from which problems are generated is affected by this transfer.

A similar point has been made by Grantham (2004, 143), who argues in favour of the importance of heuristic dependence, i.e. the notion that "theories and/or methods of a field can guide the generation of new hypotheses in a neighbouring field". Grantham does not highlight the potentially asymmetric nature of the connection. He is interested in another issue, namely that unification between disciplinary fields increases with the number of ways in which two fields are connected. He argues that heuristic dependence is one way in which unification increases. On his conception, then, unilateral problem-feeding should also increase unification, and thus potentially establish interdisciplinarity.

We think that the potentially unilateral character of problem-feeding is of special importance in sustainability science. Here we can reconsider Lenton and the tipping-points. Identifying tipping-points falls within the boundaries of Lenton's competence and academic interest. But identifying effective measures of adaptation or mitigation does not. In that sense, Lenton's work can be conceived as a case of problem-feeding without solutionfeeding. The example shows that unilateral problem-feeding can be established on the basis merely of rather weak relationships already in place between the disciplines or fields.

\subsection{Bilateral Problem-Feeding}

The $\mathrm{CO}_{2}$ example contains more bilateral problem-feeding as well: problem-feeding with solution-feeding. In the case of $\mathrm{CO}_{2}$ it was known in Arrhenius' time that the absorptive properties of the oceans were crucial to an understanding the $\mathrm{CO}_{2}$ cycle. The problem was that of providing a detailed account of these properties. Revelle and Süess advanced matters here by suggesting two mechanisms through which the oceans process $\mathrm{CO}_{2}$. It is interesting to note that the knowledge itself-especially that pertaining to the chemical properties of the ocean-was not new. Weart (2007, 7; see also his 2003) points out that the relevant reactions had been understood since the 1930s. Revelle himself was in an ideal position to tackle this issue, since he had considerable knowledge of, and experience of 
research into, oceanic chemistry and the processes by which oceanic water mixes (Weart 2007, 3f; see also his 2003).

Very often, bilateral problem-feeding is about a cognitive division of labour. One discipline or field takes on the issues of another, so that, ideally, the resolution of those issues can be transmitted back. Darden and Maull's (1977) idea of an 'interfield theory' is relevant here. An interfield theory relates one discipline or field to another by postulating, or establishing, an ontological connection between the domains of the respective disciplines or fields. Darden and Maull give four examples of ways in which disciplines or fields can be connected: one discipline may provide the physical location, the physical nature, or the structure of an entity (or process) postulated in another; or disciplines may be causally linked (see Darden and Maull 1977, 49). These four kinds of connection are not mutually exclusive. ${ }^{1}$

Interfield theories order fields ontologically, allowing for them to map onto each other. For instance, cytology and transmission genetics are interconnected through the chromosome theory of Mendelian heredity. In genetics the gene was a postulated entity corresponding to some phenotype that was inherited between generations (all-or-nothing). Cytologists provided the physical location of the gene on the chromosomes and were thus able to resolve certain issues within genetics. Thus the statistical deviation that had been noticed could be explained by the fact that proximal genes were more likely to be inherited together. Assortment is not perfectly random.

It is possible to recast Darden and Maull's case as one of bilateral problem-feeding. Transmission geneticists were in no position to investigate the physical location of the gene, as such a task lay entirely outside the scope of the methods available to them. But does this imply that the phenomenon of bilateral problem-feeding is already accounted for in the literature? No, and below we argue that it is not.

Are interfield theories necessary or sufficient, or both, for bilateral problem-feeding to ensue? Let us start with sufficiency. Suppose discipline $A$ encounters problem $P$, whereupon $P$ is fed to discipline $B$, solved through solution $S$, and $S$ is then fed back into $A$. For this to happen it seems to us that $S$ needs to be admissible in $A$. Two ways in which this could be achieved come to mind. $S$ might be, as it were, inherently admissible in $A$. For instance, the process by which $S$ was produced in $B$ could be considered to be reliable, or adequate, by standards generally applied in $A$. Alternatively, the relevant researchers in $A$ trust the ones in $B$ regardless of what the process in $B$ looks like. In neither case does the presence of an interfield theory by itself suffice. In the first, the disciplinary fields are proximate in the sense that they share epistemic standards and values beyond the interfield theory that allow them to evaluate each other's methods, tools and procedures. In the second, the interfield theory needs to be supplemented by some kind of agreement involving mutual trust. The boundaries may be ever so clear, but if the members of discipline $A$ simply do not trust members of discipline $B$ to produce reliable results it will

\footnotetext{
1 In the present paper the focus is on interdisciplinarity and hence disciplines are centre stage. Darden and Maull discuss fields. A field on, their conception is constituted by "a central problem, a domain consisting of items taken to be facts related to that problem, general explanatory factors and goals providing expectations as to how the problem is to be solved, techniques and methods, and, sometimes, but not always, concepts, laws and theories which are related to the problem and which attempt to realize the explanatory goals. A special vocabulary is often associated with the characteristic elements of a field" (Darden and Maull 1977, 44). Following Darden and Maull's own approach we will not make any particular distinction between fields and disciplines; we will generally take them to be coextensive; at the very least we will assume that a disciplinary boundary is also a field boundary (though perhaps not vice versa). Clearly, some would disagree with this: Grantham (2004) and Bechtel (1986) for instance.
} 
not help. Sustainability science is plausibly a case in point. Although various interfield theories linking social and economic phenomena to various natural phenomena (climate, ecology, etc.) are at least partly in place, mutual distrust between the disciplines sometimes hampers collaborations. The presence of an interfield theory is generally not sufficient for bilateral problem-feeding to take place.

Is an interfield theory necessary for problem-feeding to take place? When it comes to necessity the situation is somewhat different. This is largely because it is more difficult to pinpoint Darden and Maull's exact position on this matter. In order for discipline $B$ to be relevant to discipline $A$, and vice versa, some relation needs to be present. This is quite simply what it means to be relevant. But interfield theories are not just some relation. In fact, they are not even just some ontological relation. Interfield theories link the domains of disciplines in highly specific ways. We can see two ways in which interdisciplinary linking can happen that do not seem to fit the interfield theory model. First, disciplines can be linked and exchange problems in ways other than by linking their respective domains. For example, a discipline may encounter a theoretical or conceptual problem that is best thought of as one concerning its methods, theories, concepts, and so on. Suppose this problem is within the domain of that discipline, and suppose further that its solution benefits the discipline of origin. Then we shall be linking the domain of one discipline with some other aspect of another discipline. That does not seem to qualify as an interfield theory, but clearly it could be a case of bilateral problem-feeding.

Second, disciplines can be linked more loosely-i.e. in a manner less explicit or specific than happens in the case of interfield theories. Mitchell's $(2002,2003,2009)$ integrative pluralism comes to mind. Mitchell discusses composite explanations of complex phenomena, mainly within the life sciences. It is not unusual for explanations that seem to be competing (because they are mistakenly conceived of as full explanations of an explanandum) to in fact be compatible and complementary (since they add different components of the total cause). This might happen if they are integrated only at a certain level-the level of concrete particulars. At their most general level, the theories cannot be integrated to provide an account of the phenomenon in question because it is a phenomenon that has many different causes and the causal profile of specific instances may vary a lot. Here the ontological connection between the two disciplines is merely that the same phenomenon is discussed, and that (different) causes of its occurrence are investigated. This is not the kind of situation Darden and Maull have in mind, and it fails to qualify as an interfield theory. However, the upshot of the encounter can be successful bilateral problem-feeding. It is easy to conceive of situations in which partial explanations are contributed from various sources as cases of problem-feeding. It seems, especially given the first point above, that interfield theories are neither necessary nor sufficient for problem-feeding to obtain. However, this does not prevent them from being crucially important where they do occur.

\section{Barriers to Problem-Feeding, and Concept Coordination}

At this point it should be added that the transference of problems between disciplines has its obstacles.

First, there might be language barriers. The discipline of origin may formulate a problem in such a way that it cannot be understood by those in the target discipline. This might be especially visible in attempted, but failed, bilateral problem-feeding. For instance, lawyers have to decide in matters where scientific expertise is prima facie relevant. It is not uncommon for scientific experts to be consulted when, for instance, environmental risk 
management issues are being scrutinized - or when the causes of, and responsibility for, a human injury are being assessed. However, attempts at bilateral problem-feeding from law to science are not always successful in facilitating the lawyer's decision. Wahlberg (2010) reports the following expert's experience:

All right, you ask us physicians, 'What do you think?', and we write that in this particular case there are pathological changes; the changes are of such a kind that we do not regard the injury as a consequence of an accident. But the courts have during recent years always ruled in favour of the patient. I don't mind that. But then why ask us?

One reason successful bilateral problem-feeding from law to science is so difficult, Wahlberg claims, is that understandings of causation in the two fields differ. The causal concepts - or rather the ontologies corresponding to 'cause $\mathrm{law}_{\text {aw }}$ ' and 'cause science $_{\text {- }}$ are only superficially the same. For this reason it would arguably be inaccurate to say that in this example just one obstacle, a language barrier, is the problem. ${ }^{2}$ But the deployment of the term 'cause' in both fields is liable to give rise to misconceptions about the opportunities for bilateral problem-feeding. A transfer or coordination of causal concepts, or of the way in which we deploy causal terms, would facilitate this bilateral form of problem-feeding substantially.

Similarly Maull (1977) once emphasised that there needs to be some sharing of terminology if 'problem shifts' between disciplines, or disciplinary branches, are to take place. Problem shifts exemplify a special kind of bilateral problem-feeding. We have already touched on the example Maull deploys as an illustration: through a series of scientific developments, beginning around 1910, the problem of understanding the physical basis of heritable alterations shifted from genetics to biochemistry, where it was famously solved in the 1950s. Maull (1977) claims that this problem shift was associated with a transformation of the vocabulary in which the problem was formulated ensuring that this vocabulary was 'shared' by the two disciplines:

Such a 'shared' vocabulary, it turns out, can be used to identify a very special sort of problem, a problem that, although it arises within one branch of inquiry, can only be solved with the aid of another science. (Maull 1977, 144)

Sometimes, Maull claims, vocabulary is transformed in order to make problem shifts possible. 'Mutation' was first a proper term in genetics; it then referred to heritable alteration of the genotype; but later it came to stand for heritable alteration in the base sequence of DNA. This last change gave biochemists access to the expression. At the same time problems raised by mutation became difficult to solve within genetics. Though problems concerning the physical nature of the determinants of heredity arose within genetics, genetics could not solve them. One reason was methodological. Genetics deploys statistical methods and crossbreeding in order to establish regularities in heritable characteristics. However, with the transformation of the term 'mutation', the problem could shift across to biochemistry-where a solution was forthcoming. At other times, vocabulary is simply imported. However, regardless of whether vocabulary is shared because of a process of transformation (as in the mutation example) or because it has been imported, the lack of shared vocabulary is often an obstacle to the transfer of problems.

${ }^{2}$ Thus there are ontological barriers, too. At the end of Sect. 8 we refer to a methodological barrier as well. Both these types of barrier can be even more important than terminological barriers. 
At least, this holds for explicit problem-feeding. Beyond this there are more implicitperhaps even tacit-varieties of problem-feeding as well. Although these may not offer such fertile ground for philosophical discussion, they are perhaps more important than the explicit kinds of problem-feeding from a scientific perspective. Hacking $(1996,70)$ quotes a charming metaphor of James Clerk Maxwell's which speaks of the "cross-fertilization of the sciences". According to Maxwell, researchers are like honeybees, never thinking about the importance of "the dust which they are carrying from flower to flower". Some problems are perhaps never formulated in the discipline of origin, but they are nevertheless tacitly transferred to a target discipline.

Second, problems of the sort with which we are concerned can be more or less well understood (Nickles 1981; Laudan 1977). This can arise in different ways, but one issue is that scientists working in the discipline of origin sometimes have only a crude understanding of what is really constitutive of the problem they are successfully transferring (or unsuccessfully trying to transfer) to another discipline. A problem is encountered in discipline A and identified as 'belonging' to some other discipline, B. This is the reason for attempting problem-feeding. However, those working in discipline A may have unrealistic, or plainly mistaken, ideas about the solutions that can be expected in B. In sustainability science problems are frequently identified by natural scientists as belonging to a particular social science. The findings of Lenton and colleagues generate many potentially relevant problems that can be (unilaterally) transferred to the social sciences. Some of these are easier to understand from the point of view of Lenton et al. than others. Thus one might conjecture that the following two problems are rather easy to understand: What policies should be implemented to prevent us from reaching a tipping point? What are the obstacles to implementation of such policies? However, towards the end of their paper Lenton et al. call for the identification of tipping elements within the socioeconomic system. This is a much more complicated request. The attempted problem-feeding assumes that the 'socioeconomic system' can be described within the formal apparatus that produces tipping elements. It is in order to ask whether they really understand the problem that they try to transfer it.

\section{Problem-Feeding, Methodological Complementarity, and Practical Unification}

The idea of problem-feeding can usefully be connected with what Grantham (2004) calls practical unification. By contrast, many philosophical accounts of integration and unification within the sciences are founded on the idea of theoretical unification (or, as we saw in the beginning, on the idea of coordination by a higher-level concept). Even though Darden and Maull (1977) define a disciplinary field as something that only sometimes includes theories, but always includes a number of other entities - central problems, tools, methods, etc. - the actual unification they describe flows from theories about the ontologies of the disciplinary fields in question. This is why there is reason to think of their account as a modern variety of the traditional perspective on interdisciplinarity outlined in the beginning of this paper. As Grantham points out, Kincaid (1990) also emphasises theoretical interconnections in his account of unification. Kincaid's account is based on the idea that various theories can relate to each other in a number of ways, and on the corresponding idea that unification is - as it cannot be in the reductionist conception-a matter of degree. The greater the number of relations present, the more unified are the theories in question. Unity peaks when two theories are incorporated in an integrated interlevel theory-that is, a theory that unifies 
...two disparate theories by employing explanations, confirmational procedures, etc. invoking both levels, and by providing evidence that the events and entities of one theory depend upon and are constituted from those of the other. (Kincaid 1990, 590)

Let us follow Grantham in referring to the interdisciplinary consequences of these sorts of relation as theoretical unification, and let us distinguish this type of unification from practical unification. Grantham sorts methodological interactions under the latter heading, and argues that they play a more substantial role than is acknowledged in some of the alleged examples of theoretical unification deployed by Kincaid (1990). Similarly, if we return to Darden and Maull it seems true in their example, too, that practical unification is of some importance. It was in virtue of the methods and tools at their disposal that cytologists were able to conduct inquiries that were quite beyond the scope of any statistical analysis of data on the occurrence of phenotypes across generations. The interconnection between disciplines via methods appears to be important in both these cases, and methodological complementarity is - partly, at least-what is motivating the interdisciplinary encounters in them. Grantham argues that theoretical and practical unification are to some extent independent. There can be considerable theoretical unification without coordination of research practices, and there can be considerable practical unification with only a low degree of theoretical unification (Grantham 2004, 15).

Moreover, methodological complementarity (rather than theoretical integration) seems to be a necessary condition of bilateral problem-feeding-which suggests that problemfeeding entails practical unification.

The association of problem-feeding with practical rather than theoretical unification fits well with the picture we have been promoting in this paper. First, the theoretical interconnections that must be in place in order for problem-feeding to ensue can be comparatively weak. Some connection needs to be in place, but nothing as substantive as, say, an interfield theory needs to exist. Second, and trivially, partial independence implies that theoretical unification will not necessarily emerge with the advent of problem-feeding. There may be interdisciplinarity as problem-feeding without traditional interdisciplinarity.

In connection with sustainability science such results may be informative for the kind of unity that is required. Many sustainability scientists have identified integration and unification as a core problem of sustainability science. How can we integrate the models of social and natural sciences to form one, presumably cohesive, set? Maybe this ambition overshoots the target. Given the practical nature of sustainability issues-after all the research project bottoms out in tackling, or rather helping to tackle, problems the solutions to which will always be judged by actual outcomes-it is perhaps perfectly in order to see problem-solving, as opposed to other epistemic ideals, as the overriding aim. In this approach theoretical and conceptual unification should primarily be sought only to the extent that it is instrumental in solving these problems.

\section{Problem-Feeding as a Driver of Concept and Method Coordination}

We have already touched on Grantham's view that unification increases with a rise in the number (or variety) of ways in which two fields are connected. He also claims that unification is enhanced by an increase in the significance of the connections that are already in place. A connection becomes more significant if it begins to transform the neighbouring field. That is, if the introduction of a novel concept, generalization, 
technique, or heuristic leads to considerable change in the absorbing field, then the change is regarded as significant and unification is advanced (Grantham 2004, 144).

We have just claimed that there may be interdisciplinarity as problem-feeding without traditional interdisciplinarity. But an argument in favour of the idea that problem-feeding is especially important for interdisciplinarity should also provide reasons for thinking that problem-feeding is significant in the sense that it might begin to transform the neighbouring field.

We have claimed that sustainability science is an interdisciplinary field, and that the disciplines involved in the exchange of problems are located either side of the natural/ social science divide. Sustainability science differs in many respects from the cases that have caught the attention of the majority of philosophers of interdisciplinarity. Most philosophical treatments concentrate on disciplinary fields that are in many respects proximate. They share much at the outset, and this makes the sharing and shifting of problems a lot smoother. Within sustainability science this is decidedly not the case-at least, when it comes to integrating the natural and social dimensions of sustainability. However, and this is our next point, this does not undermine the recognition that problems need to be transferred.

Our suspicion is that the need to solve problems by first feeding them to another field is sometimes itself the fundamental reason why other kinds of bridge between distinct disciplinary fields are created. We have already seen how this may generate changes in, or transfers of, vocabulary in the example given by Maull (1977). She shows how the need to problem-feed might result in shared vocabulary. Problem-feeding sometimes stimulates concept coordination. A degree of conceptual transfer from $A$ to $B$, or coordination between them, normally results in the possibility that problems in $B$ can be fed into and (sometimes) solved in $A$. When this happens it would be misconceived to consider problem-feeding as the outcome of other forms of integration. Quite the contrary, it is the starting point. It begins to transform the neighbouring field.

We would like to conclude this sketch of the ways in which problem-feeding can drive other kinds of transfer or coordination by saying a few words about method transfer and method coordination. Method transfer can occur in several ways. Methods can be migrated domain-only-wise where a method already known within one discipline is deployed to extract information from a domain to which it has not previously been applied. From within the ranks of ecology there has been a degree of optimism about the power of the methods used there. This transfer does not have to result in actual interdisciplinarity, since the other field operating in the domain need not be influenced by it. However, disciplinary method transfer can result in the migrating method out-competing the methods that were previously in play. Two observations by Ronald Coase (see also Mäki 2009) are interesting in this context:

[I]n the long run it is the subject matter, the kind of question which the practitioners are trying to answer, which tends to be the dominant factor producing the cohesive force that makes a group of scholars a recognizable profession... However, in the short run, the ability of a particular group in handling certain techniques of analysis, or an approach, may give them such advantages that they are able to move successfully into another field or even to dominate it. (Coase 1978, 204)

More often, perhaps, method transfer generates situations in which methods are somehow used in concert. The transfer results in methodological pluralism. 'Mixed methods' research is one possible result. But the label 'methodological pluralism' collects several distinct varieties of the type. Some problem-feeding events are such that several distinct 
methods are used more or less in sequence to solve various linked problems. At least one non-sequential variety is similar: certain phenomena have multiple causes whose investigation is carried out using different methods. A third kind of methodological pluralism that might result from method transfer is illustrated by the use of multiple methods to obtain results that are robust. In this case methodological pluralism involves the use of a set of disparate methods to achieve an epistemic end: for example, solving a particular problem complex (or chain), or checking results for robustness.

Disciplinary method transfer or coordination consorts well with our account of interdisciplinarity, especially since cases of such transfer or coordination do not always constitute interdisciplinarity (unlike cases of transfer or coordination motivated by problemfeeding concerns, which do). Simply sharing a certain method is not always sufficient for interdisciplinarity, even if the method has migrated from the one field to another. For instance, statistical analysis is widespread in both the natural and the social sciences. However, this does not seem to warrant talk of interdisciplinarity. Why is this? Is it because the migrating method is insufficiently anchored in the field from which it is being transferred? This would be alarming news to those involved in the many unificationist programmes being prosecuted today. Philosophers trying to facilitate the unification of $A$ and $B$ would risk finding that the very fact that unification was provided by methods (or concepts, or problems, or some such) suggested by a third party prevents interdisciplinarity between $A$ and $B$. The risk should not be exaggerated, of course; further developments between $A$ and $B$ would stand a better chance of being truly interdisciplinary.

In our opinion the most interesting interdisciplinary cases of method transfer typically centre on problem-solving processes. In an early contribution to the modern literature on interdisciplinarity, Sherif and Sherif (1969) discuss a case that helps to bring this out:

Yet when Dr William Schottstaedt and his colleagues studied the detailed biochemical and physiological records of patients in a metabolic ward, they found that variations in these strictly physiological measures of metabolism were significantly related to the vicissitudes of interpersonal relationships among patients, with nurses and doctors, and with visitors. (Sherif and Sherif 1969, 6)

A little further on, the authors continue:

If we would start to inquire further why these particular patients were hospitalized for metabolic disorders, and not others, we would find ourselves immediately in problems requiring demographic study of different populations and in problems requiring institutional analysis of admittance procedures and financing; and these might very well lead us to problems of the political and economic systems in which the institutions functions (ibid.).

In pursuing the problems that arise from this observation Dr Schottstaedt and his colleagues would have ventured beyond their immediate expertise. This could happen in at least two ways. One is simply to import the methods and approaches necessary to address the questions one might have. As Sherif and Sherif seem to imply, however, this might be quite untenable in the long run. Prima facie it would be more rational to engage in problem-feeding and export the problem to a discipline where the methods and tools are readily available. Problem-feeding does not always result in method transfer. Sometimes we can either export the problem or import the know-how (the method, in this case). Doing one of the two is enough. What problem-feeding does require, minimally, is that some methodological coordination takes place. (To the extent that this has not been settled a problematic methodological barrier is in place.) 


\section{Conclusion: The Basic Evolutionary Ladder Revisited}

We have argued that problem-feeding is a salient type of interdisciplinary collaboration in sustainability science. We have also argued that it cannot be accounted for by the traditional perspective, since it does not necessarily involve coordination by higher level concept. We have placed problem-feeding in context of recent discussion, particularly the work of authors like Grantham, Kincaid, Mitchell, Darden, and Maull.

The interdisciplinarity resulting from problem-feeding between researchers can be local and temporary and does not require collaboration between proximate disciplines. By contrast, to make good sense of traditional integrative interdisciplinarity we must arguably associate it with a longer-term, global form of close, interdisciplinary collaboration.

Furthermore, whether or not one believes in the interdisciplinary evolutionary ladder (Jantsch 1970/1972, 15) it seems to us that a basic step can consist of problem-feeding. This can occur where interdisciplinarity starts to evolve. The step is not a prerequisite of reaching further steps of the traditional ladder, such as coordination by "higher-order concept" or method. Such coordination may be secured by an alternative route, for different reasons. In this sense we are pluralists. But it is clear that a preference for bilateral problem-feeding-especially-may trigger the coordination of concepts and methods. Problem-feeding already introduces the sense of purpose the traditional perspective locates at more advanced steps of the interdisciplinary ladder. Jantsch, for one, thought that a sense of purpose was introduced when a common axiomatics for a group of related disciplines was defined at the next higher hierarchical level (Jantsch 1970/1972, 16).

It appears to us that the very fact that the sense of purpose is introduced so early on in interdisciplinarity as problem-feeding yields more opportunities for local interdisciplinarity, i.e. interdisciplinary encounters motivated by a local problem. Perhaps several cases that have been thought of as Mode 2 (Gibbons et al. 1994) can be reformulated as cases of this type. However, we are not suggesting the two conceptions are inter-translatable. Indeed there is an important difference between them. Gibbons et al. $(1994,29)$ talk about Mode 2 as something requiring a "homogenised theory or model pool". But problem-feeding does not presuppose a homogenised theory or model pool, and we have argued in the paper that unilateral problem-feeding does not require anything like that either. Successful bilateral problem-feeding requires more coordination, but there is still a significant difference.

Acknowledgments We want to thank Lennart Olsson, Paul Robinson, Cecilia and Jep Agrell, Stefan Schubert, and two anonymous reviewers of this journal for a number of constructive comments on an earlier version of this manuscript. This work was supported by the Linnaeus programme LUCID ("Lund University Centre of Excellence for Integration of Social and Natural Dimensions of Sustainability" (www.lucid.lu.se/), FORMAS, 2008-2018). An early forerunner of the text was presented at the Third Biennial Conference of the Society for Philosophy of Science in Practice (June 22-24, 2011; Exeter, UK) and has been published on Phil Sci Archive as: Thorén, Henrik and Persson, Johannes (2011) Philosophy of Interdisciplinarity: Problem-Feeding, Conceptual Drift, and Methodological Migration. http://philsci-archive.pitt.edu/8670/.

Open Access This article is distributed under the terms of the Creative Commons Attribution License which permits any use, distribution, and reproduction in any medium, provided the original author(s) and the source are credited.

\section{References}

Apostel, L., Berger, G., Briggs, A., \& Michaud, G. (Eds.). (1972). Interdisciplinarity: Problems of teaching and research in universities. Paris: Organization for Economic Cooperation and Development. 
Arrhenius, S. (1896). On the influence of carbonic acid in the air upon the temperature of the ground. Philosophical Magazine and Journal of Science, 41, 237-276.

Bechtel, W. (1986). The nature of scientific integration. In W. Bechtel (Ed.), Integrating scientific disciplines (pp. 3-52). Dordrecht: Nijhoff.

Bolin, B., \& Eriksson, E. (1959). Changes in the carbon dioxide content of the atmosphere and sea due to fossil fuel combustion. In B. Bolin, (Ed.), The atmosphere and the sea in motion (pp. 130-142). New York: The Rockefeller Institute and Oxford University Press.

Coase, R. (1978). Economics and contiguous disciplines. Journal of Legal Studies, 7, 201-211.

Craig, H. (1957). The natural distribution of radiocarbon and the exchange time of carbon dioxide between atmosphere and sea. Tellus, 9, 1-17.

Darden, L., \& Maull, N. (1977). Interfield theories. Philosophy of Science, 44, 43-64.

Edlund, C., Hermerén, G., \& Nilstun, T. (1986). Tvärskap. Lund: Studentlitteratur.

Gibbons, M., Limoges, C., Nowotny, H., Schwartzman, S., Scott, P., \& Trow, M. (1994). The New Production of Knowledge: The Dynamics of Science and Research in Contemporary Societies. London: Sage.

Grantham, T. (2004). Conceptualizing the (dis)unity of science. Philosophy of Science, 71, 133-155.

Hacking, I. (1996). The disunities of the sciences. In P. Galison \& D. J. Stump (Eds.), The disunity of science (pp. 37-74). Stanford: Stanford University Press.

Jantsch, E. (1970/1972). Inter- and transdisciplinary university: A systems approach to education and innovation. (Originally published in Policy Sciences 1970). Higher Education, 1, 7-37.

Jerneck, A., et al. (2011). Structuring sustainability science. Sustainability Science, 6, 69-82.

Kincaid, H. (1990). Molecular biology and the unity of science. Philosophy of Science, 57, 575-593.

Klein, J. T. (1990). Interdisciplinarity. History, theory and practice. Detroit: Wayne State University Press.

Kuhn, T. (1969/1970). 'Postscript'. In His: The structure of scientific revolutions (2nd ed.). Chicago: University of Chicago Press.

Laudan, L. (1977). Progress and its problems: Towards a theory of scientific growth. Berkeley, Los Angeles: University of California Press.

Lenton, T., Held, H., Kriegler, E., Hall, J., Lucht, W., Rahmstorf, S., et al. (2008). Tipping elements in the earth's climate system. PNAS, 105, 1786-1793.

Mäki, U. (2009). Economics imperialism: Concept and constraints. Philosophy of the social sciences, 39, $351-380$.

Maull, N. (1977). Unifying science without reduction. Studies in History and Philosophy of Science, 8, $143-162$.

Mitchell, S. D. (2002). Integrative pluralism. Biology and Philosophy, 17, 55-70.

Mitchell, S. D. (2003). Biological complexity and integrative pluralism. Cambridge: Cambridge University Press.

Mitchell, S. D. (2009). Unsimple truths: Science, complexity, and policy. Chicago: University of Chicago Press.

Nagel, E. (1961). The structure of science. London: Routledge.

National Academy of Sciences, National Academy of Engineering, and Institute of Medicine. (2005). Facilitating interdisciplinary research. Washington, DC: National Academies Press.

Nickles, T. (1981). What is a problem that we may solve it? Synthese, 47, 85-118.

Nowotny, H., Scott, P., \& Gibbons, M. (2001). Rethinking science: Knowledge and the public in an age of uncertainty. London: Sage.

Persson, J., \& Sahlin, N.-E. (2013). Vetenskapsteori för sanningssökare. Stockholm: Fri Tanke Bokförlag.

Revelle, R., \& Süess, H. (1957). Carbon dioxide exchange between atmosphere and ocean and the question of an increase of atmospheric CO2 during the past decades. Tellus, 9, 18-27.

Sherif, M., \& Sherif, C. (Eds.). (1969). Interdisciplinary relationships in the social sciences. Chicago: Aldine Publishing Company.

Thorén, H., \& Persson, J. (2011). Philosophy of interdisciplinarity: Problem feeding, conceptual drift, and methodological migration. PhilSci-Archive. http://philsci-archive.pitt.edu/8670/.

Wahlberg, L. (2010). Legal questions and scientific answers: Ontological differences and epistemic gaps in the assessment of causal relations. Ph.D. thesis, Lund University.

Weart, S. (1997). Global warming, cold war, and the evolution of research plans. Historical Studies in the Physical and biological Sciences, 27, 319-356.

Weart, S. (2003). The discovery of global warming. Cambridge, Mass: Harvard University Press.

Weart, S. (2007). Roger Revelle's discovery. American Institute of Physics. http://www.aip.org/history/ climate/pdf/Revelle.pdf. Accessed 17 May 2011. 